\title{
Wie Ausbildungsbetriebe soziale Ungleichheit reproduzieren: Der Ausschluss von Migrantenjugendlichen bei der Lehrlingsselektion ${ }^{1}$
}

\author{
Christian Imdorf
}

Published as:

Imdorf, Christian (2010). Wie Ausbildungsbetriebe soziale Ungleichheit reproduzieren: Der Ausschluss von Migrantenjugendlichen bei der Lehrlingsselektion. In: H.-H. Krüger, U. Rabe-Kleberg, R.-T. Kramer \& J. Budde (eds.), Bildungsungleichheit revisited. Bildung und soziale Ungleichheit vom Kindergarten bis zur Hochschule. Wiesbaden: VS Verlag für Sozialwissenschaften, 263-278.

DOI 10.1007/978-3-531-92201-0_14

Original source of publication: http://www.springerlink.com/content/gl71g74377921223/

The final publication is available at www.springerlink.com

Anschrift des Autors:

Seminar für Soziologie der Universität Basel

Petersgraben 27

4051 Basel

Schweiz

E-Mail: christian.imdorf@unibas.ch

Tel. +416126728 82

Fax. +41612672820

\footnotetext{
Der vorliegende Aufsatz wurde anlässlich zweier Forschungsaufenthalte am Laboratoire d'Économie et de Sociologie du Travail (Aix en Provence) sowie an der Adam Smith Research Foundation (University of Glasgow) durch ein Stipendium des Schweizerischen Nationalfonds ermöglicht. Er gibt an mehreren Stellen Auszüge aus einer ausführlicheren Publikation zu Migrantenjugendlichen in der betrieblichen Ausbildungsplatzvergabe wieder (vgl. Imdorf 2008) und erweitert zugleich deren theoretische Argumentation.
} 


\section{Das Tor zur betrieblichen Ausbildung als Produktionsort sozialer Ungleichheit}

Die duale Berufsausbildung mit ihren beiden Lernorten Schule und Betrieb ist traditionell in den deutschsprachigen Berufsbildungssystemen stark verankert. In der Schweiz stehen über 200 verschiedene Ausbildungsberufe zur Wahl, deren Zertifikate den Eintritt in den qualifizierten Arbeitsmarkt regulieren, wobei die Arbeitsmarktchancen von Personen ohne berufliche Qualifikation in den letzten zwei Jahrzehnten abgenommen haben (vgl. Meyer 2009). Auch wenn eine abgeschlossene Berufslehre noch keine sichere Arbeitsstelle garantiert, so gilt sie noch immer als wichtige Voraussetzung dafür. Die berufsrelevante Humankapitalvermittlung sowie der Erwerb eines beruflichen Titels sind dabei zwei wichtige Erträge der beruflichen Erstausbildung, die vor Jugendarbeitslosigkeit, prekären Berufskarrieren und den damit verbundenen Merkmalen sozialer Benachteiligung und Ungleichheit schützen (vgl. Imdorf 2008).

Ein gelingender Übergang in die berufliche Ausbildung entscheidet demnach über die späteren beruflichen Möglichkeiten der Ausbildungsanwärter. Da ein beruflicher Abschluss ohne einen Ausbildungsplatz nicht zu haben ist, beginnt die Integration in den Arbeitsmarkt als eine wichtige Bedingung für gesellschaftliche Anerkennung und soziale Sicherheit bereits mit der erfolgreichen Lehrstellensuche. Dies trifft in besonderem Maße für die Vermittlung von Arbeitsmarktchancen von ,Ausländern' zu. Die Berufsausbildung stellt auch für sie den Zugang zu den qualifizierten Segmenten des Arbeitsmarkts sicher und leistet somit einen bedeutenden Beitrag zu ihrer gesellschaftlichen Integration (vgl. Seibert 2005, S. 232).

Die betriebliche Erstausbildung als Tor in den qualifizierten Arbeitsmarkt ist im deutschsprachigen Raum jedoch gerade für ausländische Schulabgänger nicht in gleichem $\mathrm{Maß}$ zugänglich wie für Inländer (für die Schweiz vgl. Hupka/Stalder 2004; Imdorf 2005; für Deutschland: Boos-Nünning/Granato 2008, S. 59; Diehl/Friedrich/Hall 2009; für Österreich: Wieser et al. 2008, S. 37). Auch die deutsche Bundesregierung (2007, S. 70) hält im Nationalen Integrationsplan inzwischen fest, dass die Ausgangsposition von Migrantenjugendlichen für einen Übergang in die berufliche Ausbildung deutlich schlechter ist als jene von Jugendlichen ohne Migrationshintergrund. Damit wird auf höchster politischer Ebene ein soziales Problem anerkannt, welches sich in Deutschland und der Schweiz in den vergangenen Jahren verschärft hat (vgl. Granato 2006), auf das aber bereits seit Ende der Neunzigerjahre hingewiesen wurde (Attia, et al. 2000, S. 71).

Am stärksten vom tendenziellen Ausschluss aus der betrieblichen Berufsausbildung betroffen sind insbesondere jene Jugendlichen, die im öffentlichen Diskurs als ,Ausländer' gelten (vgl. Diehl/Friedrich/Hall. 2009). In Deutschland handelt es sich dabei um türkischstämmige Jugendliche und um Zuwanderer aus den Nachfolgestaaten der Sowjetunion (ebd.); in der Schweiz sind es dagegen in erster Linie Jugendliche aus Familien, die aus den Nachfolgeländern des ehemaligen Jugoslawien in die Schweiz immigriert sind (vgl. Imdorf 2008). Männliche Jugendliche aus diesen Gruppen haben dabei besonders große Probleme, Zugänge in die betriebliche Berufsausbildung zu erhalten (vgl. Diehl et al. 2009). 
Als mögliche Gründe für den Ausschluss von Migrantenjugendlichen von der Berufsausbildung werden verschiedene Erklärungen vorgebracht. Dominant ist der Diskurs um die für eine berufliche Ausbildung ungenügende schulische Qualifizierung und (insbesondere sprachliche) Leistungsfähigkeit von Jugendlichen, die als ausländisch gelten (vgl. Imdorf 2007). Des Weiteren wird auf unzureichende Kenntnisse der Mechanismen und Gepflogenheiten, die den Lehrstellenmarkt prägen, sowie auf einen reduzierten Zugang $\mathrm{zu}$ innerbetrieblichen Rekrutierungsnetzwerken bei Migrantenfamilien hingewiesen (vgl. Lee/Wrench 1983; Boos-Nünning/Granato 2008; Meyer 2009). Schließlich ist im Nationalen Integrationsplan auch von „Vorbehalten bei der Einstellungsentscheidung” die Rede (Bundesregierung 2007, S. 70), womit auf betriebliche Rekrutierungs- und Selektionsstrategien verwiesen wird, welche Migrantenjugendliche möglicherweise benachteiligen (vgl. Boos-Nünning/Granato 2008; Imdorf 2008).

Inzwischen liegen Befunde vor, welche die Schuldefizitthese in der Tendenz widerlegen. Neuere Untersuchungen zeigen, dass Jugendliche mit Migrationshintergrund noch bei Kontrolle ihres schulischen Leistungsausweises erheblich reduzierte Chancen auf einen betrieblichen Ausbildungsplatz haben (vgl. Boos-Nünning/Granato 2008, S. 70f; Diehl et al. 2009; Imdorf 2007). Auch anderweitige individualtheoretische Argumente, die eine unzureichende Bildungsplanung und nachteilige Bildungsorientierungen bzw. Suchstrategien von Migrantenjugendlichen und ihrer Eltern behaupten, konnten empirisch bisher nicht belegt werden (vgl. Boos-Nünning/Granato 2008). In Anbetracht der Untersuchungsergebnisse von Diel et al. (2009) gilt es zudem auch die Bedeutung der bewerberseitigen Netzwerkressourcen für die Ausbildungsplatzvergabe kritisch zu hinterfragen.

Angesichts der anhaltenden Übergangsprobleme von ausländischen Schulabgängern in die Berufsausbildung und der damit einhergehenden Hypothek nicht nur für die Betroffenen selbst, sondern vor allem auch für die Kommunen, denen in letzter Instanz die Bearbeitung der daraus entstehenden sozialen Probleme zufällt (vgl. Imdorf 2008), stellt sich die Frage nach einer adäquaten theoretischen Konzeptualisierung der Eintrittshürden in die betriebliche Ausbildung. Der vorliegende Aufsatz plädiert dafür, die bisherigen individualtheoretischen Erklärungen um betriebliche Dimensionen zu erweitern, um den Ausschluss von jungen ,Ausländern' bei der Lehrlingsselektion zu analysieren. Wir gehen davon aus, dass erst eine betriebliche Perspektive ein angemessenes Verständnis ihres Ausschlusses bei der Selektion und damit eine wirkungsvolle Unterstützung ihrer Integration in Ausbildung, Arbeitsmarkt und Gesellschaft ermöglicht.

\section{Theoretische Zugänge zum Ausländerausschluss bei der Stellenbesetzung}

\subsection{Lehrlingsselektion: Ein für Diskriminierung prädestinierter Spezialfall der Personalselektion}

Obwohl Auszubildende im dualen System sowohl im Betrieb als auch in der Berufsschule 
qualifiziert werden, sind es im deutschsprachigen Raum die Betriebe, die oft in eigener Regie über den Abschluss eines Ausbildungsvertrags entscheiden. Bei der betrieblichen Auswahl von Auszubildenden handelt es sich dabei um einen Spezialfall der Personalselektion, der sich aus folgenden Gründen für die Untersuchung von Diskriminierung besonders gut eignet: Während die innere Gestaltung der Ausbildungsverhältnisse staatlich reguliert ist, gilt dies für die Auswahl der Lernenden selbst kaum. Die Schweiz kennt keine Antidiskriminierungsgesetze, auf die sich bei der Lehrlingsselektion Benachteiligte berufen könnten. Betriebe erhalten zudem meist eine hohe Zahl von Bewerbungen für eine offene Ausbildungsstelle, und dies eröffnet ihnen eine große Wahlfreiheit bei der Entscheidung für einen Kandidaten, der absehbar den eigenen Ansprüchen am besten zu genügen vermag. Da sich in der Schweiz die große Mehrheit der Bewerber aus Abgängern der obligatorischen Schule sowie des Übergangssystems zusammensetzt, und da deren persönliches Entwicklungspotential inmitten der Adoleszenz als kaum berechenbar gilt, stehen den Betrieben kaum Informationen zur Verfügung, welche die Produktivität eines Lernenden abschätzbar machen würden. Um eine Prognose hinsichtlich ihrer betrieblichen und beruflichen Eignung zu stellen, wenden Klein- und Mittelbetriebe - betriebliche Ausbildung findet besonders häufig in Klein- und Mittelbetrieben statt, die in der Schweiz annähernd $90 \%$ sämtlicher Ausbildungsplätze anbieten (vgl. Imdorf 2008, S. 153) - zudem nur limitierte Ressourcen auf. Den Ausbildungsverantwortlichen solcher Betriebe fehlt es meist an spezifischen Fachkompetenzen der Personalauswahl, und ihnen steht für die Aufgabe der Lehrlingsselektion nur wenig Zeit zur Verfügung. Sie bedienen sich daher mehr oder weniger effektiver, im Wesentlichen auf Erfahrung basierende Lösungen der Komplexitätsreduktion. All diese Merkmale lassen erwarten, dass Diskriminierung bei der Ausbildungsstellenvergabe - verstanden als Personalselektion, die über eine meritokratische Beurteilung individueller Leistungsmerkmale hinausgeht - eher der Normalfall als ein Sonderfall ist. Die Analyse der Auswahl von Lernenden in Klein- und Mittelbetrieben bietet sich entsprechend als Ort der Produktion von sozialer Ungleichheit in der Ausbildungslaufbahn im Speziellen sowie als Untersuchungsgegenstand von Diskriminierung bei der Stellenvergabe im Allgemeinen an.

\subsection{Modelle zur Erklärung des Ausländerausschlusses bei der Personalselektion}

Betriebliche Selektionsmechanismen, die über soziale Netzwerkrekrutierung und die Bewertung von Schulzeugnissen hinausgehen, wurden in den bisher vorliegenden quantitativen Untersuchungen nur unzureichend reflektiert und allenfalls als ungeklärte ,Residualgröße' in Betracht gezogen. Mit Ausnahme vereinzelter Studien zu Großbetrieben (vgl. Bommes 1996; Moser 2004), finden sich kaum Untersuchungen, die das betriebliche Entscheidungsverhalten bei der Vergabe von Ausbildungsstellen vertieft analysieren. Studien, die auch Klein- und Mittelbetriebe in die Analyse des Ausländerausschlusses bei der Lehrlingsselektion miteinbezogen haben, sind älteren Datums (Lee/Wrench 1983; Schaub 1991). Da diese Studien nur beschränkt die Theoriebildung zur betrieblichen 
Auswahl von Auszubildenden stimuliert haben, scheint es vorerst sinnvoll, in der bestehenden Forschungsliteratur nach Erklärungen für den empirisch beobachtbaren Ausländerausschluss bei der Personalselektion zu suchen.

Unzureichendes Humankapital (vgl. Becker 1993) sowie fehlende soziale Netzwerke (vgl. Granovetter 1995, S. 133; Holzer 1987) stellen auch in der internationalen Literatur die dominanten Konzepte dar, um nachteilige Arbeitsmarktzugänge von Ausländer- bzw. Migrantengruppen zu erklären. Zahlreiche Bewerberexperimente (vgl. Pager 2007), die es erlauben, Humankapital und soziale Netzwerkressourcen zu kontrollieren, haben jedoch gezeigt, dass sich der Ausländerausschluss mit den zwei obgenannten Erklärungsansätzen nicht befriedigend verstehen lässt. Obwohl solche Feldexperimente die Messung von Diskriminierung bei der Stellenvergabe ermöglichen, vermögen sie jedoch nicht zu erklären, weshalb Arbeitgeber motiviert sind, Kategorien der sozialen Zugehörigkeit (neben Nationalität, Ethnie oder Rasse auch Geschlecht, Alter oder Behinderung) bei der Personalauswahl zu verwerten.

Die angloamerikanische Diskriminierungsforschung bietet zu dieser Frage eine breite Palette von Antworten an (für einen Forschungsüberblick vgl. Pager/Shepherd 2008). Am meisten verbreitet sind wohl jene Ansätze, welche aus Arbeitgebersicht danach fragen, welche Bewerber zukünftig am wenigsten produktiv sein könnten. Sie basieren auf der Annahme, dass alle Bewerber mit einer individuellen Produktivität bzw. mit individueller Kompetenz ausgestattet sind, die sich unter idealen Bedingungen objektiv messen ließe (vgl. Eymard-Duvernay 2008, S. 56). Das Modell der statistischen Diskriminierung (vgl. Arrow 1972; Phelps 1972) hebt dabei den betrieblichen Informationsmangel über diese individuelle Produktivität von Bewerbern hervor, bzw. die Kosten, welche zu deren Messung anfallen würden. Die Arbeitgeber lösen dieses Problem, indem sie generalisierte Produktivitäts-Annahmen über ,Ausländer' machen, die sich durch Erfahrung oder anderweitige empirische Belege stützen lassen. Implizite Vorurteilsmodelle (vgl. Quillian 2006) betonen dagegen tiefere Produktivitätserwartungen von Arbeitgebern gegenüber Ausländergruppen, welche sich eher auf Gefühl und Glauben als auf empirische Belege stützen.

Das Problem solcher Erklärungsansätze besteht jedoch darin, dass die Reduktion von Bewerbern auf Annahmen über ihre individuelle Produktivität die komplexen sozialen Bezüge und Abhängigkeiten eines Betriebs vernachlässigt, die ebenso zum wirtschaftlichen Erfolg beitragen (vgl. Kirschenman/Neckerman 1991, S. 231). In den Blick gerät damit zum einen das soziale Beziehungsgeflecht innerhalb eines Produktionsbetriebs, d.h. die horizontalen Sozialbeziehungen innerhalb der Belegschaft. Modelle der sozialen Schließung (vgl. Roscigno/Garcia/Bobbitt-Zeher 2007) behaupten, dass Diskriminierung bei der Stellenvergabe vorwiegend durch partikularistische Interessen der Belegschaft vor Ort verursacht sei. Die Personalverantwortlichen respektieren solche Partikularinteressen, wenn sie nach (inländischen) Personen suchen, die sozial möglichst gut in eine bestehende inländische Belegschaft ,passen' (vgl. Jenkins 1986), um damit künftigen sozialen Spannungen möglichst vorzubeugen. Zum anderen sind die Vorgesetzten aber auch daran interessiert, solche Bewerber einzustellen, von denen $\mathrm{zu}$ erwarten ist, dass sie sich der 
betrieblichen Anleitung und Führung weder entziehen noch widersetzen, die sich also möglichst reibungsfrei ,managen' lassen. ,Ausländer' können dann bei der Personalauswahl benachteiligt sein, wenn ihre betriebliche Fügsamkeit und ihr Wille zur Unterordnung zur Diskussion stehen (vgl. Waldinger/Lichter 2003, S. 143f). Aus dieser Perspektive lässt sich Produktivität als betrieblicher Selektionskalkül eher als Resultat von Sozialbeziehungen am Arbeitsplatz und weniger als Funktion individueller Kompetenzen im Sinne der Humankapitaltheorie verstehen (vgl. Shih 2002, S. 102).

Neben den innerbetrieblichen horizontalen und vertikalen Sozialbeziehungen gilt es schließlich auch die betrieblichen Markt- und Kundenbeziehungen bei der Analyse von Diskriminierung zu berücksichtigen. Sowohl Produktions- als auch Dienstleistungsbetriebe sind in der Regel gewillt, die Bedürfnisse und Wünsche der Kundschaft bei wichtigen Entscheiden zu berücksichtigen. Gemäß Becker (1971, S. 75ff) kann die Kundschaft bei der Bewertung eines Produkts oder einer Dienstleistung Merkmale des Verkaufspersonals wie deren Rassen- oder Religionszugehörigkeit mitberücksichtigen und aus rassistischen Gründen eine Verkaufsabteilung meiden.

Die Berücksichtigung dieser Sozialbeziehungen, von denen die Koordinationsleistungen des betrieblichen Produktionsprozesses entscheidend abhängen, verweist auf eine genuin soziologische Theorie der Personalauswahl, die versucht, die wohlbekannten kognitiven und sozialpsychologischen Prozesse bei der Personalselektion mit organisationalen Kontexten und Opportunitätsstrukturen in Beziehung zu setzen. Aus dieser Perspektive gilt es, den (Ausbildungs-)Betrieb als zentrale Analyseeinheit bei der Theoretisierung von Diskriminierungsprozessen zu berïcksichtigen, und die betrieblichen Mechanismen herauszuarbeiten, welche eine askriptive Selektion erst ermöglichen (vgl. Baron/Pfeffer 1994; Reskin 2003).

\section{Multiple Koordinationsanforderungen an Auszubildende: Ein organisationstheoretisches Modell der Lehrlingsselektion}

Im Folgenden wird eine organisationstheoretisch inspirierte und um eine Theorie der Rechtfertigung erweiterte Konzeption der Ausbildungsplatzvergabe vorgeschlagen, um den Ausschluss von ,Ausländern' aus Sicht der Betriebe zu verstehen. Die Konzeption wurde in Konfrontation mit Rechtfertigungsreden von Ausbildungsverantwortlichen in kleineren Schweizer Betrieben entwickelt. ${ }^{2}$

\footnotetext{
Die empirischen Forschungen wurden im Rahmen der Untersuchung ,Lehrlingsselektion in KMU' von 2004-2006 am Heilpädagogischen Institut der Universität Fribourg durchgeführt. Die Studie wurde im Rahmen des Nationalen Forschungsprogramms ,Integration und Ausschluss' (NFP51) durch den Schweizerischen Nationalfonds gefördert. Die Zitate im vorliegenden Text basieren auf leitfadenstrukturierten Interviews mit Ausbildungsverantwortlichen aus 81 Klein- und Mittelbetrieben der Deutschsprachigen Schweiz aus dem Jahr 2005. Bei den Betrieben handelte es sich um private Arzt- und Zahnarztpraxen, Autogaragen und Autospritzereien, Schreinereien sowie um kaufmännische Abteilungen in Betrieben unterschiedlicher Branchen. Die betriebliche Stichprobe ist das Resultat einer Nachverfolgung von erfolglosen Bewerbungen Jugendlicher auf ausgewählte Ausbildungsplätze (Dentalassistentin, Medizinische Praxisassistentin, Auto-
} 


\subsection{Auf der Suche nach akzeptablen und akzeptierten Auszubildenden}

Ausgangspunkt sind zunächst Bedürfnisse und Sachzwänge von Ausbildungsbetrieben, die sich aus dem Zwang des ökonomischen Überlebens auf dem Branchenmarkt ergeben (für eine ausführlichere Darstellung der nachfolgenden organisationstheoretischen Überlegungen siehe Imdorf 2008, S. 119ff). Bei der Lehrlingsauswahl geht es daher immer auch um ein potentielles ,Profitgeschäft' (vgl. Mühlemann, Wolter, Fuhrer/Wüest 2007): Die Ausbildung muss sich für einen Betrieb früher oder später lohnen, also zum eigenen Bestand beitragen.

Ein Hauptproblem, wenn Betriebe Auszubildende auswählen, besteht darin, unter Bedingungen von Zeitknappheit und Unsicherheit das implizite, erfahrungsbasierte betriebliche Ausbildungsstellenprofil (u.a. Anforderungen der beruflichen, arbeitsförmigen und sozialen Passung) auf pragmatische Weise mit den gegebenen jugendlichen Bewerbern - bzw. was von ihnen aufgrund externer Unterscheidungsangebote der Betriebsumwelt gewusst werden kann - so zu ,matchen', dass dieser Selektionsprozess nicht in der Umwelt eines Betriebs in Frage gestellt wird. Mehrfache Strukturprobleme im Selektionsprozess (u.a. multidimensionale Anforderungen des Stellenprofils, die kein Bewerber in ihrer Gesamtheit annähernd perfekt erfüllt) führen dazu, dass ,der beste Kandidat' gar nicht gefunden werden kann. Profitmaximierung als Leitprinzip bei der Lehrlingsauswahl funktioniert daher nicht, und Betriebe brechen ihre Suchbemühungen frühzeitig ab, sobald ein Kandidat gefunden ist, der die multiplen betrieblichen Anforderungen, genügend befriedigt' (im Sinne des satisficing, vgl. March/Simon 1958, S. 169).

Darüber hinaus erfordert dieses pragmatische matching von Bewerber und Stelle, dass das Resultat der Selektion in den betriebsrelevanten Öffentlichkeiten auf Akzeptanz stößt. Das Publikum eines Kleinbetriebs setzt sich einerseits aus der Kundschaft und den Geschäftspartnern zusammen. Andererseits gilt es Protest innerhalb der eigenen Mauern, insbesondere unter den Mitarbeitern, die in kleineren Betrieben meist an den Auswahlverfahren mitbeteiligt sind, zu vermeiden, um deren Kooperation und Arbeitsmotivation zu sichern (vgl. Boltanski/Chiapello 2003).

\subsection{Koordinationsanforderungen als Rechtfertigungsressourcen: Die legitime Bewertung von Bewerbern im Hinblick auf ein Gemeinwohl}

Um die Komplexität und Mehrdeutigkeit bei der Personalselektion erfolgreich zu bewältigen, sind die Selektionsverantwortlichen daher auf Formen und Ordnungen der Rechtfertigung angewiesen, die es erst ermöglichen, eine getroffene Wahl gegenüber den betrieblich relevanten Öffentlichkeiten als legitim darzustellen. Personalentscheide lassen

mechaniker und -monteur, Autolackierer, Schreiner, sowie kaufmännische Ausbildungen). Die Studie erhebt keinen Anspruch auf Repräsentativität, sondern dient hier primär der empiriegeleiteten Entwicklung eines theoretischen Modells der Ausbildungsplatzvergabe mit dem Ziel, eines ihrer Resultate - den praktischen Ausschluss von Migrantenjugendlichen - besser zu verstehen. 
sich rechtfertigen, wenn die Selektionskriterien in diesen Öffentlichkeiten als einem Gemeinwohl dienend und somit als fair und gerecht erachtet werden (vgl. EymardDuvernay 2004, S. 98). Das bedeutet, dass ein Selektionsentscheid nicht nur den Eigeninteressen der Angehörigen des Betriebs oder der Kundschaft zugute kommen darf, sondern darüber hinaus auch einem übergeordneten Gemeinwesen. ${ }^{3}$

So lässt sich beispielsweise eine Selektionslogik, die primär auf betriebliche Produktivität ausgerichtet ist, damit legitimieren, dass letztere über das betriebliche Wohl hinaus - vermittelt etwa über betriebliche Sozialabgaben und steuerliche Leistungen - auch einem übergeordneten gesellschaftlichen Wohl zugute kommt. Alternativ lässt sich ein Betrieb auch eingebettet in ein lokales Gemeinwesen denken (z.B. ein Dorf, in welchem ein Betrieb angesiedelt ist). Der soziale Zusammenhalt dieses Gemeinwesens kann unter anderem dann gefördert werden, wenn die Ausbildner bei der Bearbeitung von Problemen mit Auszubildenden durch deren Eltern unterstützt werden. Im (Familien-) Unternehmen agiert ein Lehrmeister am Arbeitsplatz dann als eine Art Vaterersatz, der über die Unterstützung der leiblichen Eltern des Lehrlings in seiner Autorität zusätzlich gestärkt wird, um letzteren zu maßregeln (vgl. Boltanski/Chiapello 2003, S. 182). Die antizipierte Elternunterstützung kann auf diesem Hintergrund als legitimes Selektionskriterium vertreten werden.

Entsprechend können unterschiedliche Selektionslogiken unterschieden werden, die sich jeweils in spezifischer Weise auf ein Gemeinwesen und auf ein daran geknüpftes Allgemeinwohl beziehen lassen. ${ }^{4}$ Von selektionsrelevanter Bedeutung ist nun, dass die Beförderung eines Gemeinwohls eine bestimmte Form der Koordinierung (vgl. Boltanski/Thévenot 2007, S. 95f) bzw. der Koordination ${ }^{5}$ von sozialen Akteuren voraussetzt, damit sich im betrieblichen Alltag die dazu nötigen Verhaltensweisen aufeinander abstimmen lassen (vgl. Eymard-Duvernay 2004, S. 66). ${ }^{6}$ Einer Person, die in

3 Wir beziehen uns nachfolgend auf das Konzept der Rechtfertigungsordnung, das von Boltanski und Thévenot 1991 in ihrem schulbildenden Werk ,De la justification. Les économies de la grandeur' (deutsch: Boltanski/Thévenot 2007) eingeführt wurde. In ihrem Werk leiten die Autoren unterschiedliche Formen von Gemeinwesen und Gemeinwohl aus mehreren klassischen Werken der politischen Philosophie her (vgl. Boltanski/Thévenot 2007, S. 97). Sie beziehen sich unter anderem auf die modellhafte politische Marktphilosophie von Adam Smith (2006 [1790]), der mit der Argumentation, dass der Wohlstand der Reichen allen zugute komme, die marktwirtschaftliche Ordnung rechtfertigen konnte.

4 Das übergeordnete Gemeinwohl als konstitutive Bedingung einer Rechtfertigungsordnung ist sowohl in den politischen Philosophien (vgl. Boltanski/Thévenot 2007, S. 115) als auch im eigenen empirischen Material schwierig nachzuweisen, es bleibt in der Regel implizit. Die angeführten zwei Beispiele sind konstruiert und empirisch nicht gedeckt. Entscheidend ist, dass sich ein persönlicher Gewinn oder Vorteil (hier: ein aus betrieblicher Sicht vertretbarer Selektionsentscheid) erst dann überzeugend rechtfertigen lässt, wenn dieser auf einer höheren Ebene gleichzeitig einer Gemeinschaft zugute kommt, was dann deren Gemeinwohl befördert (ebd., 111).

5 Auf dem Werk von Boltanski und Thévenot gründet auch die französische Konventionen-Ökonomie (vgl Eymard-Duvernay 2004, S. 71ff), von welcher wir den Begriff der Koordination übernehmen.

6 Es lässt sich zeigen, dass von solchen verschiedenen Formen bzw. Konventionen der Koordination Boltanski und Thévenot (2007) beziehen sich darauf, wenn sie von Ordnungsprinzipien sprechen - auch die Funktionstüchtigkeit und damit der Bestandeserhalt eines Betriebs abhängt, weshalb die vorliegende Konzeption der Personalauswahl den Anspruch einer Organisationstheorie hat, die über eine bloße Theorie der Rechtfertigung hinausgeht. 
einer betrieblich relevanten Situation Koordinationsfähigkeit unter Beweis stellt, wird Größe (grandeur) in Bezug auf diese Situation zugesprochen (vgl. Boltanski/Thévenot 1999, S. 363).

Boltanski und Thévenot (2007, S. 183) sprechen nun mit Bezugnahme auf die Bewertung von Größe in unterschiedlichen Gemeinwesen von verschiedenen Welten, in denen ein je spezifisches Koordinations- bzw. Ordnungsprinzip (u.a. Effizienz oder Seniorität) zugrunde gelegt wird (verstanden auch als moralisches Prinzip bzw. Gerechtigkeitsprinzip einer jeweiligen Welt). Solche Ordnungsprinzipien funktionieren wie Äquivalenzprinzipien, die es gestatten, in Respektierung des jeweilig gültigen Prinzips Wertigkeiten von Menschen festzulegen. Im Kontext der Personalselektion gestattet der Rekurs auf solche Prinzipien den Personalverantwortlichen, zwei oder mehrere Bewerber begründbar miteinander zu vergleichen, zu bewerten und in eine Rangordnung zu bringen.

Die Wertigkeiten eines Bewerbers werden im Rahmen der Lehrlingsselektion in einer Reihe unterschiedlicher Prüfsituationen - u.a. bei der Sichtung von Bewerbungsunterlagen, im Rahmen von Leistungstests oder Betriebspraktika - mittels so genannter Bewährungsproben bestimmt. Solche Bewährungsproben (zum Begriff vgl. Boltanski/Chiapello 2003; für empirische Beispiele vgl. Imdorf 2008) ermöglichen - mittels einer begründbaren differenzbasierten Auslese von Personen auf Rangplätze unterschiedlicher Wertigkeit - die Reduktion von Komplexität und Ungewissheit im Selektionsprozess. Bei der Personalauswahl stützt sich eine Bewährungsprobe auf Selektionsformen und -routinen unterschiedlichen Formalisierungsgrads, wobei sehr verschiedene Diagnose-Instrumente zum Einsatz kommen: Neben den wohlbekannten Methoden der Leistungs- und Kompetenzmessung stellt auch der ,Bauch' (als Metapher für das affektive Evaluationsinstrumentarium des Betriebs) ein zentrales Prüfinstrument dar. Die fehlende soziale Passung eines, Ausländers' wird nicht objektiv gemessen, sondern durch die Selektionsbeteiligten systematisch gefühlt.

Überträgt man nun also die zentralen Ideen bei Boltanski und Thévenot (2007) auf die betriebliche Auswahl von Auszubildenden, so haben die Selektionsverantwortlichen multiple aber dennoch limitierte Normen der Koordination (und entsprechende Äquivalenzprinzipien) zur Verfügung, um Bewerber in eine Rangordnung zu bringen und den Selektionsentscheid zu rechtfertigen. Jede Koordinationsnorm verweist auf eine eigene ,Welt'. Man kann nun verschiedene solcher Welten wie die ,industrielle Welt', die ,häusliche Welt', die ,projektförmige Welt' oder die ,Welt des Marktes' unterscheiden, um den Ausländerausschluss bei der Selektion von Auszubildenden besser zu verstehen.

\section{Lehrlingsauswahl in den verschiedenen,Welten' eines Betriebs \\ 4.1 Lehrlingsselektion in der industriellen Welt}

In der ,industriellen Welt' eines Betriebs wird Handelsware produziert oder eine Dienstleistung angeboten - Produkte, deren Bereitstellung effiziente Produktionsabläufe erfordern, die ein Auszubildender möglichst nicht stören sollte. In der industriellen Welt 
zählen dabei neben den traditionellen Arbeitstugenden (wie Pünktlichkeit, Regelmäßigkeit oder Ordnung im Sinne industrieller Koordinationsanforderungen) berufsspezifische und vor allem schulische Kompetenzen. Betriebe streben als industrielle Welt ein möglichst günstiges Kosten-Nutzen-Verhältnis an: Den betrieblichen Aufwänden für ein Ausbildungsverhältnis - Lehrlings- und Ausbildnerlöhne (bzw. die Zeit, die für die Anleitung der Auszubildenden erforderlich ist), Personaladministration, Anlagen und Materialien - steht ein potentieller Nutzen gegenüber, der sich in Lohneinsparung für durch Lehrlinge substituierte (un)qualifizierte Arbeitskraft sowie längerfristig - im Falle einer

Übernahme des Ausgebildeten - in Einsparungen von Rekrutierungs- und Einarbeitungskosten für qualifizierte Arbeitskraft niederschlägt (vgl. Mühlemann et al. 2007). In dieser industriellen Welt des Betriebs wird den als ausländisch geltenden Ausbildungsanwärtern bei der Selektion insbesondere ein Mangel an schulischen und sprachlichen Kompetenzen zugeschrieben. Dabei fällt auf, dass sich die Betriebe mit diesen Argumenten primär auf die industrielle Welt der Berufsschule (bzw. auf deren Leistungsund Koordinationsanforderungen) beziehen, und weniger auf jene des eigenen Betriebs. Befürchtet wird, dass ,schulische und sprachliche Defizite' die erfolgreiche Absolvierung der Berufsschule sowie den Abschluss der Lehrabschlussprüfung in Frage stellen könnten mit der Folge, dass die betrieblichen Rentabilitätserwartungen an das Ausbildungsverhältnis nicht eingelöst würden. Bei den angeführten Argumenten handelt es sich jedoch oftmals um in sich nicht sehr schlüssige Rechtfertigungen, und sie scheinen zudem vielfach einer erfahrungsbasierten Stützung zu entbehren (vgl. Imdorf 2008, S. 135f). ${ }^{7}$

\subsection{Die Anforderungen der häuslichen Welt eines Ausbildungsbetriebs}

Die Koordinationsanforderung der ,häuslichen Welt' eines Betriebs scheinen besonders relevant zu sein, um die Diskriminierung von ,Ausländern' bei der Lehrstellenvergabe jenseits von defizitären sprachlichen und schulischen Leistungszuschreibungen $\mathrm{zu}$ verstehen (vgl. Imdorf 2008, S. 138ff): Gemeint sind solche zwischenmenschlichen Beziehungen und Erwartungen zwischen den Mitarbeitern, die sich in Klein- und Mittelbetrieben an den Koordinationsformen der traditionellen, patriarchal strukturierten Familie orientieren: Vertrauen, Achtung und Respekt, die Differenz zwischen ,Wir und den Anderen', nach Alter geordnete Autoritäts- und Abhängigkeitsverhältnisse, traditionell

7 Genau besehen kann Sprache als das ,kostengünstigste' Argument der Rechtfertigung von Selektionsentscheidungen betrachtet werden, denn aufgrund seiner unmittelbaren Plausibilität erübrigen sich weitere Stützungen durch empirische Belegerzählungen. Das Sprachargument ist für die Betriebe aber vor allem auch ,kostengünstig', weil es jeden potentiellen, gegen den Ausschluss von ,ausländischen' Bewerbern gerichteten Protest frühzeitig und effizient abzuwenden erlaubt. Denn Sprache ist ein wohlfeiles Argument, welches seinen Halt im Common Sense hat und durch seine ,Unschlagbarkeit' überzeugt: Wer nicht sprachkompetent ist, kann die mit einer Lehre verbundenen Leistungsanforderungen nicht erfüllen. Die Betriebe bedürfen keiner Belege dafür, dass die Ortssprachkompetenzen eines multilingualen Jugendlichen den Anforderungen einer Lehre nicht genügen würden. Der Verweis auf Sprachdefizite bei ,Ausländern' ist selbsterklärend und sieht keine zuverlässigen betrieblichen Bewährungsproben vor (vgl. Imdorf 2008, S. 136). 
ausgerichtete Geschlechterrollen. Im betrieblichen Kontext schlägt sich dies in sozialen Kontrollstrukturen, im Respekt vor den Vorgesetzten, der Anerkennung der Autorität älterer Mitarbeiter oder der geschlechtsspezifischen Arbeitsteilung nieder. Darauf beziehen sich die Mitglieder eines Betriebes oftmals als ,Geist des Hauses' und Bewerber werden daraufhin beobachtet, ob sie ,dazu passen'. In dieser häuslichen Welt ist die Wertigkeit einer Person durch eine Vertrauenshierarchie bestimmt, die von Tradition, Abstammung, sozialer Nähe und Respekt getragen ist (vgl. Boltanski/Thévenot 1999, S. 370) und die Beziehungen zwischen Personen strukturiert. Entsprechend besteht die Bewährungsprobe oft aus einem ,Vertrauenstest' (vgl. Thévenot 2001, S. 415) , aus dem Bauch' und auf der Basis von gegenseitigem Kennen und Anerkennen.

,Ausländer' finden in diesem Familienmodell kaum einen Platz. Sie gelten als Fremde, die den ,Geist des Hauses' in Frage stellen, und mit ihrer Präsenz droht die Beeinträchtigung der betrieblichen Funktionstüchtigkeit beziehungsweise der Anfall hoher , sozialer Kosten' (vgl. Sayad 2006, S. 35ff.) im eigenen Haus. Die Ausbilder betonen auch ohne Bezugnahme auf eine antizipierte Sprachproblematik die Privilegierung von ,Einheimischen' (im Sinne von Nicht-,Ausländern'), da diese besser in das soziale Gefüge des eigenen Betriebs passten. Dabei wird das vertrauensbasierte Inländerprivileg im Auswahlverfahren oftmals bereits bei der Vorselektion auf der Grundlage der vorliegenden Informationen zu Namen und Nationalität im Bewerbungsdossier durchgesetzt.

Die Rechtfertigungsreden im eigenen Material verweisen wiederholt darauf, dass die soziale Zusammensetzung der Belegschaft von hoher betrieblicher Relevanz ist. Bei ausländischen Bewerbern werden teilweise soziale Konflikte am Arbeitsplatz und die Gefahr von Mobbing antizipiert. In einem größeren zahnmedizinischen Betrieb hat ein für die Vorselektionen zuständiger Verwalter argumentiert, dass die relativ vielen Bewerbungen von ,Ausländerinnen', „und eben teilweise, ja eben von Ex-Jugoslawien” für den Betrieb ein „Riesenproblem” seien, da ,in Serbien, Montenegro, Kosovo (...) gewisse Kulturen aufeinander prallen" würden. Gestützt auf diese alltagsbewährte Kulturkonfliktthese bildet der Betrieb bevorzugt ,Schweizerinnen' zu Dentalassistentinnen aus. Ein Zahnarzt einer anderen Praxis berichtete über eine Konflikteskalation in seinem interethnisch zusammengesetzten Team von beträchtlicher betrieblicher Bedeutsamkeit. Er verwies auf eine bis dahin noch nicht erlebte Dimension von Mobbing zwischen seinen Angestellten mit ,katastrophalen' Auswirkungen auf die Arbeitsprozesse. Diese Konflikterfahrung, deren Ursache vom Zahnarzt auf religiös kulturell bedingte Unterschiede zurück geführt wurde, hatte Konsequenzen für seine anschließende Auswahl neuer Auszubildender:

„Wir haben den Anspruch Team. Ich muss schauen, was braucht unsere Praxis im Moment (...). Ich bin überhaupt nicht ausländerfeindlich, aber.. Das hat ein sehr unglückliches/Spannungen ausgelöst in dieser Praxis, und ich habe gesagt, ich will ganz sicher niemanden mehr aus diesem Kreis jetzt in dieses bestehende Team einbauen. Weil ich (...) weitere Konflikte dann gesehen habe, die auf uns zukommen.“

Das Argument des zitierten Zahnarztes, dass er selbst „nichts gegen Ausländer habe”, dass er aber zur Vorbeugung betrieblicher Probleme gegen ,Ausländer' entscheiden müsse, 
kehrt auch in anderen Aussagen wieder. Ein Garagist verweist darauf, dass seine Mitarbeiter einen , ausländischen' Lehrling möglicherweise nicht positiv aufnehmen würden:

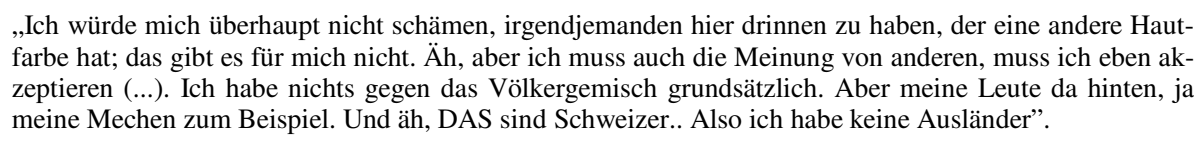

„Ich würde mich überhaupt nicht schämen, irgendjemanden hier drinnen zu haben, der eine andere Hautfarbe hat; das gibt es für mich nicht. Äh, aber ich muss auch die Meinung von anderen, muss ich eben akzeptieren (...). Ich habe nichts gegen das Völkergemisch grundsätzlich. Aber meine Leute da hinten, ja meine Mechen zum Beispiel. Und äh, DAS sind Schweizer.. Also ich habe keine Ausländer”.

Wie die abschließende Sequenz aus einer Arztpraxis zeigt, sitzt die Befürchtung, dass die ,Ausländer' den intimen, familiären Zusammenhalt in der Praxis gefährden könnten, tief in den ,Bäuchen' betrieblicher Mitarbeiter:

Arzthelferin: „Also bei mir war es nur Sympathie, ich wollte sie [eine als ausländisch geltende junge Frau, die sich ohne Erfolg in der Arztpraxis beworben hatte] eigentlich nicht, von Anfang nicht (...). Eben dieses Überbeflissene, das hat mich genervt, (...) und einfach ihre ganze Art, die sie hatte, hat mich (...) Schwierig zu sagen. JA [Stimme wird lauter], aber ich weiß, es ist schwierig (...). Ja, es ist dann eben aus dem Bauch heraus, dass man nur schwer sagen kann (...). Sie war mir vielleicht irgendwie fremd gewesen mit ihrer ganzen Art (...). Nur schon wie sie geredet hat, oder wie sie gefragt hat (...). Wir sind eigentlich eine Praxis, die sehr intim arbeitet, oder, also die, sagen wir einmal, äh, wenn Sie mit den Lehrtöchtern, sobald Sie hier sind als Lehrtochter, sind wir per DU, oder, und äh ja, wir sind so eben, angenehm vom Klima her".

Im Unterschied zur ,Anpassungsfähigkeit' von ausländischen Jugendlichen, die in den Interviews relativ selten problematisiert wurde, scheint der Selektionskalkül der ,sozialen Passung' bzw. der ,Teampassung' ein wesentlicher Mechanismus für den betrieblichen Ausländerausschluss zu sein.

Während harmonische Arbeitsbeziehungen für die Führbarkeit eines produktiven Arbeitsteams indirekt bedeutsam sind, verweisen anderweitige Argumente direkt auf den betrieblichen Bedarf nach führbaren und fügsamen Auszubildenden. So wird beispielsweise darauf geachtet, dass nicht mehrere bzw. zu viele Mitarbeitende derselben, als ausländisch geltenden Nationalität rekrutiert werden. Damit lässt sich die Bildung fremdsprachlich homogener Mitarbeitergruppen vermeiden, deren Kommunikation und Dynamik schwierig zu kontrollieren wäre. Bei , ausländischen Jugendlichen' werden zudem öfter die elterlichen Unterstützungsressourcen im Hinblick auf die Lösung betrieblicher Probleme gering geschätzt. Die Ausbilder wünschten sich jedoch, bei der Durchsetzung ihrer ,häuslichen Autorität' zur Sanktionierung eines aus betrieblicher Sicht problematischen Verhaltens eines Auszubildenden auf die Unterstützung der Eltern zählen zu können. Schließlich sind junge , ausländische' Männer in besonderem Maße von betrieblicher Problemantizipation betroffen, insbesondere wenn ihnen ein machohaftes Verhalten zugeschrieben wird. Sie stehen dann im Verdacht, dass sie sich gegenüber weiblichen Vorgesetzten nicht angemessen unterordnen könnten.

\subsection{Kundenbindung als betriebliches Kalkül bei der Lehrlingsselektion}


Zusätzlich zu ihrer ,industriellen' und ,häuslichen Größe' erhalten Auszubildende im Direktkontakt mit Kunden einen zusätzlichen Wert für den Betrieb, da sie den Verkauf eines Produktes oder einer Dienstleistung fördern oder hemmen können (vgl. Imdorf 2008, S. 137f). Durch ihre Erscheinung und durch ihr Verhalten stoßen Auszubildende bei der Kundschaft in unterschiedlichem Maß auf Gefallen, was sich auf aktuelle und zukünftige Kundenbindungen auswirken kann. In den Worten eines Garagisten, der neu auf weibliche Auszubildende setzt: „Wir sind eine Frauengarage, und ich habe das Gefühl, eine Frau in der Werkstatt bringt mir noch mehr Kunden". Arbeitgeber repräsentieren somit die Bedürfnisse ihrer eigenen Klientel, wenn sie im Selektionsprozess die für die Kundenbindung nötigen Koordinationsressourcen prüfen. Dazu zählen verkaufsrelevante Kriterien wie sympathisches oder hübsches Aussehen, gute' Umgangsformen, sowie vorteilhafte Sprach- und Sprechweisen. Der betriebliche Wert eines Mitarbeiters im Austausch mit der Kundschaft basiert dabei auf unterschiedlichen Typen der Koordination bzw. - im Sinne von Boltanski und Thévenot (2007, S. 367ff) - auf einem ,Kompromiss' von mehreren Rechtfertigungsordnungen, insbesondere wenn die Qualität der Kundenbindung von adäquaten ,Sozialkompetenzen' der Mitarbeiter abhängt.

Kundenbindungen lassen sich erstens in der häuslichen Welt verorten, sofern sie eine harmonische persönliche Beziehung voraussetzen, was in kleineren Betrieben üblich und im Falle von personalisierten Dienstleistungen (u.a. in Arzt- und Zahnarztpraxen) fundamental ist. Zweitens kann die Verhaltensanforderung an Mitarbeitende, mit adäquaten kommunikativen und Kontakt fördernden Kompetenzen Beziehungen mit Kunden zu knüpfen, über die häuslichen Normen hinaus als eine eigene Größe verstanden werden. Die entsprechende Welt haben Boltanski und Chiapello (2003) als ,projektbasierte Polis' bezeichnet, in der ,Netzwerkmenschen' soziale Beziehungen projektförmig leben. In einer Zahnarztpraxis wurde beispielsweise mit Bezugnahme auf diese projektförmige Welt berichtet, dass sich ausländische Bewerberinnen in Praktika verschlossen gezeigt und sich zurückgezogen hätten, was aus Sicht der Personalverantwortlichen als „Handicap” und als den Patienten nicht zumutbar beurteilt wurde.

Betriebe können drittens Vorbehalte haben, weil sie befürchten, dass ,ausländische' Auszubildende Kundenbindungen aufgrund von Kundenvorbehalten gegenüber ausländischen Angestellten gefährden könnten. Im Direktkontakt mit der Kundschaft nehmen sie dann einen eigentlichen Warencharakter an, und jenseits ihrer sozialen Kompetenzen rückt ihre Größe in einer, Welt des Marktes' in den Blick. In einem kleinen Reisebüro argumentierte die Inhaberin aus Rücksicht auf ihre Klientel, dass sie sich nicht vorstellen könne, eine Kopftuchtragende junge Frau einzustellen, da sie vom Klatsch der eigenen Kunden her wisse, dass diese „völlig irgendwie gegen solche Ausländer etwas haben“. Ein Zahnarzt äußerte das Gefühl, „dass gute, langjährige Schweizerpatienten“ aufgrund ausländischer Namen von Angestellten ,abspringen könnten“. Ausbilder, die Bewerber aufgrund eines zugeschriebenen ,Marktwerts' beurteilen, bewegen sich jedoch auf brüchigem Boden der Rechtfertigung, da die Bewerber die Zuschreibungsprozesse und damit ihre Größe in der marktförmigen Welt nicht beeinflussen können. Deshalb handelt es sich hier gemäß Boltanski und Thévenot (2007, S. 116ff) um eine illegitime Rechtfertigungsordnung, die 
sich nicht auf ein Gemeinwohl berufen kann und protestanfällig ist. Dies könnte ein Grund dafür sein, dass Kundenvorbehalte gegenüber ausländischen Jugendlichen in den Interviews selten direkt thematisiert wurden.

Dagegen fand sich interessanterweise in mehreren Betrieben das Argument, dass man bei der Lehrlingsauswahl auch darauf achte, dass neue Auszubildende keine unliebsame Kundschaft anziehen. Eine Zahnarztpraxis befürchtete beispielsweise, sich mit der Einstellung einer albanischen Lehrtochter für albanische Patienten, welche ,keinen Sinn für eine normale Zahnhygiene“ hätten, attraktiv zu machen. Ein Garagist problematisierte die Kundschaft der Albaner und Kosovaren („Das sind unmögliche Typen”). Er verwies auf den eigenen Gebrauchtwagenmarkt,

„wo wir sie haben und zu kämpfen haben mit. Wir hatten einmal einen [kosovarischen Angestellten] im Waschbereich hinten, und dann hatten wir zehn, zwölf nachher ums Haus herum, und da sagten wir, das müssen wir nicht haben (...). Und dann sagten wir einfach einmal generell, keine Ausländer, also in diesem Sinne, keine Ausländer, einfach vor allem Kosovo, und die Bewerbungen waren neunzig Prozent solche".

Dieser Selektionskalkül verweist auf eine unerwartete Spielart der Marktlogik: ,Ausländische' Jugendliche können offenbar die marktförmige Welt eines Betriebs stören, indem sie eine aus betrieblicher Sicht unliebsame ethnische Klientel anziehen, die dem Betrieb besondere Probleme bereiten könnte. Um dies zu vermeiden, verzichten einige Ausbildungsbetriebe drauf, Jugendliche mit entsprechender ethnischer Herkunft einzustellen. Dies erscheint dann als Erfolg versprechende betriebliche Strategie, um die Nachfrage nach den eigenen Produkten bzw. Dienstleistungen im Eigeninteresse zu beschränken.

\subsection{Ausländerausschluss in multiplen Welten}

Aus dem eigenen empirischen Material ergeben sich vielfältige Hinweise darauf, dass es bei der Lehrlingsselektion nicht darum geht, den leistungsstärksten Kandidaten zu ermitteln. Die Ausbildungsbetriebe suchen vielmehr nach einer Person, welche die unterschiedlichen Koordinationsanforderungen in den verschiedenen Welten eines Betriebs befriedigend erfüllt. Eine Lehrstelle zu besetzen, bedeutet für den Ausbildungsbetrieb, ,Kosten und Nutzen' - Ausbilder sprechen oftmals in der Metapher des gegenseitigen ,Geben und Nehmens' - in allen relevanten Welten des Betriebs abzuwägen und das Störpotential, das Auszubildende in diese verschiedenen Welten hineintragen könnten, zu erkennen und möglichst rechtzeitig abzuwenden (vgl. Imdorf 2008, S. 124). ${ }^{8}$ Bewerber um einen Ausbildungsplatz sind entsprechend gefordert, im Rahmen des Auswahlverfahrens

\footnotetext{
Bei den skizzierten Welten handelt es sich - vor jeder ökonomischen Verwertungs- und Überlebenslogik um historische und soziale Konstruktionen (vgl. Boltanski/Thévenot 1999, S. 369). Ausgehend von der Prämisse, dass Betriebe auch bei Verzicht auf ökonomische Profitmaximierung grundsätzlich überleben können, erscheint es letztlich unmöglich, den wirtschaftlichen Beitrag von sozialen gegenüber ökonomischen Faktoren der betrieblichen Reproduktion zu quantifizieren (u.a. da sich die sozialen Faktoren einer ökonometrischen Vermessung entziehen).
} 
ihr Einfügungsvermögen in diese verschiedenen Welten des Betriebs glaubhaft zu machen. Die Lehrlingsselektion kann dabei als eine komplexe Verkettung von zeitlich aufeinander folgenden oder simultan ablaufenden Bewährungsproben in den verschiedenen Welten verstanden werden, welche ein erfolgreicher Kandidat zu bestehen hat.

Vor diesem Hintergrund erweist sich der Ausschluss der ,Ausländer' bei der Lehrstellenvergabe als Resultat einer für sie verhängnisvollen Verknüpfung von Anforderungen, welche die Ausbildungsbetriebe an die Betroffenen stellen, und die sie weniger wahrscheinlich als ihre Konkurrenten erfüllen können (ebd., 145): Ausländische Jugendliche gelten aus der Perspektive der Ausbilder in den relevanten Welten des Betriebs als potentiell nicht ,passend' bzw. als , sperrig': Ihre Kompetenz steht in der industriellen Welt der Berufsschule in Frage, was die erfolgreiche Absolvierung der Berufslehre gefährden kann; ihre Binnenloyalität in der häuslichen Welt, in der sie als potentielle Störer einer eingespielten sozialen Ordnung wahrgenommen werden; und ihre Kundenfreundlichkeit auf dem Branchenmarkt, wo sie ein Kundenrisiko darstellen, u.a. weil sie Risikokunden anziehen.

Das empirische Material der eigenen Untersuchung verweist entsprechend darauf, dass von als ausländisch geltenden Jugendlichen in besonderem Maße betriebliche Störungen erwartet werden. Das behauptete Problempotential, welches diese Jugendlichen in die Betriebe hineintragen könnten, geht dabei über Belange der industriellen Welt, auf welche die Probleme von ausländischen Lehrstellensuchenden und ihre institutionelle Bearbeitung oft reduziert werden, weit hinaus. Das skizzierte theoretische Modell der Ausbildungsstellenvergabe erklärt dabei nur die primären Mechanismen des Ausländerausschlusses. Daran schließen anderweitige sekundäre Ausschlussprozesse an, welche die Chancen, einen Ausbildungsplatz zu erhalten, für ausländische Lernende zusätzlich reduzieren. Denn aufgrund ihres primären Ausschlusses müssen sie sich im Gegensatz zu den inländischen Bewerbern nun häufiger, über größere geographische Distanzen, über längere Dauer im Jahresverlauf, sowie auf ein breiteres berufliches Spektrum bewerben. Dies führt sie in einen eigentlichen Teufelskreis, da solche Merkmale aus betrieblicher Sicht unabhängig des Ausländerstigmas als zusätzliche Indizien für absehbare Probleme im Ausbildungsverlauf gedeutet werden (näher dazu Imdorf 2008, S. $143 \mathrm{ff})$.

\section{Schlussfolgerungen}

Versteht man Betriebe als Organisationen, die in ihrem alltäglichen Prozessieren verschiedene Koordinations- und Rechtfertigungsordnungen miteinander in Einklang bringen müssen, dann bietet es sich an, das Phänomen der ,Ausländer'-Diskriminierung bei der Personaleinstellung als Konsequenz der Handhabung miteinander konfligierender Normen der Koordination - und damit unterschiedlicher Gerechtigkeitsnormen - in verschiedenen betrieblichen Welten zu deuten (vgl. Imdorf 2008, S. 126). Diskriminierung bei der Stellenvergabe lässt sich nun verstehen als eine Ungleichbehandlung von 
Bewerbern trotz vergleichbarer industrieller Größe bzw. Kompetenz, unter anderem wenn askriptive soziale Merkmale (u.a. Nationalität, Geschlecht, Alter) in den betrieblichen Entscheidungsprozessen mitverwertet werden. Solche sozialen Selektionskalküle erscheinen in solchen Betrieben als funktional, die bei der betriebsinternen Einschätzung der Bedingungen des eigenen Bestanderhaltes noch vor den Anforderungen der industriellen Welt den Bedürfnissen der häuslichen, projekt- und marktförmigen Welten den Vorrang geben. Es sind die Anforderungen dieser Welten, welche durch ihre je eigenen Reproduktionsanforderungen und Zutrittskriterien unablässig sozial ungleiche Zugänge zu betrieblichen Ausbildungsplätzen produzieren und damit soziale Ungleichheit im Übergang von der Schule in die Berufsausbildung reproduzieren. Diskriminierung ist somit das Resultat von befürchteten Problemen in diesen Welten, jenseits einer Problemantizipation in der industriellen Welt.

Die Nicht-Berücksichtigung ,ausländischer' Schulabgänger bei der Ausbildungsplatzvergabe hat also ihre Grundlage in der Funktionsweise der Betriebe selbst, in ihrer Handhabung der Herausforderungen der für sie relevanten Welten, deren Zugang den Betroffenen verwehrt wird. Neben den bisher vorgestellten Koordinations- und Rechtfertigungsordnungen könnte eine weitere von Boltanski und Thévenot (1999, S. 372) aufgezeigte Welt, in welche Betriebe unabdingbar eingebunden sind - die ,staatsbürgerliche Welt' - allenfalls Mittel bereithalten, um dem Ausländerausschluss bei der Stellenvergabe vorzubeugen. Diese Welt reklamiert einen Gesellschaftsvertrag, gegründet auf einem Kollektivinteresse, der das eigensüchtige Interessehandeln der Betriebe durch Bindung an das Kriterium der Wahrung des sozialen Friedens Beschränkungen unterwirft (vgl. Imdorf 2008, S. 126). Die staatsbürgerliche Norm erfordert von den Betrieben zuallererst die Einhaltung des Gesetzes sowie die Respektierung sozialer und gewerkschaftlicher Rechte. Da der Staat aber die betriebliche Lehrlingsselektion kaum reguliert, interveniert die staatsbürgerliche Welt bei der Vergabe von Ausbildungsplätzen nur schwach. Im staatsbürgerlichen Gleichheitsbegriff verankerte soziale Gerechtigkeitsvorstellungen werden auch von den Betrieben nicht in genügendem $\mathrm{Ma} ß$ auf freiwilliger Basis respektiert, obwohl dies in einzelnen Fällen durchaus gegeben ist. Solange die Vergabe von Ausbildungsplätzen staatlich nicht geregelt werden kann, braucht es somit Interventionen außerhalb der staatsbürgerlichen Welt, um die betrieblichen Ausbildungschancen von ausländischen Jugendlichen zu erhöhen.

Das hier vorgeschlagene Verständnis des betrieblichen Ausländerausschlusses beruht auf einem soziologischen Modell der Ausbildungsstellenvergabe. Es ist zuallererst ein theoretischer Entwurf, der es ermöglicht, Ausbildungsbetriebe als bildungspolitisch relevanten und bearbeitbaren Ort der Produktion von Bildungsungleichheit zu verstehen. Der Entwurf kann vorerst dazu dienen, dokumentierte empirische Rechtfertigungsreden von Ausbildungsverantwortlichen zum Ausschluss von ausländischen Ausbildungsplatzsuchenden (vgl. Imdorf 2008; Lee/Wrench 1983; Schaub 1991) angemessen zu deuten. Praxisrelevante Implikationen der hier vorgestellten Überlegungen stehen jedoch unter dem Vorbehalt ihrer weiteren empirischen und wissenschaftlichen Bewährung (zu möglichen praktischen Implikationen des Modells vgl. Imdorf 2008, S. 
146ff). Dazu sind Arbeitgeberbefragungen notwendig, deren Erhebungsinstrumente die Ideen des vorliegenden theoretischen Entwurfs aufgreifen.

\section{Literatur}

Arrow, K. J. (1972): Models of Job Discrimination. In: Pascal, A. H. (Hrsg.): Racial Discrimination in Economic Life. Lexington: D.C. Heath. 83-102

Attia, I./Aziz, L./Marburger, H./Menge, J. (2000): Auf Ausbildungsplatzsuche. In: Attia, I./Marburger, H. (Hrsg.): Alltag und Lebenswelten von Migrantenjugendlichen. Frankfurt a. M.,.71-100

Baron, J. N./Pfeffer, J. (1994): The Social Psychology of Organizations and Inequality. In: Social Psychology Quarterly, Jg. 57, H. 3. 190-209

Becker, G. S. (1993): Human capital: A theoretical and empirical analysis with special reference to education. Third Edition. New York

Becker, G. S. (1971): The Economics of Discrimination. Second Edition. Chicago: University of Chicago Press

Boltanski, L./Chiapello, E. (2003): Der neue Geist des Kapitalismus. Konstanz

Boltanski, L./Thévenot, L. (1999): The Sociology of Critical Capacity. In: European Journal of Social Theory, Jg. 2, H. 3. 359-377

Boltanski, L./Thévenot, L. (2007): Über die Rechtfertigung. Eine Soziologie der kritischen Urteilskraft. Hamburg

Bommes, M. (1996): Ausbildung in Grossbetrieben. Einige Gründe, warum ausländische Jugendliche weniger Berücksichtigung finden. In: Kersten, R./Kiesel D./Sargut, S. (Hrsg.): Ausbilden statt Ausgrenzen. Jugendliche ausländischer Herkunft in Schule, Ausbildung und Beruf. Frankfurt. 31-44

Boos-Nünning, U./Granato, M. (2008): Integration junger Menschen mit Migrationshintergrund: Ausbildungschancen und Ausbildungsorientierung. Forschungsergebnisse und offene Fragen. In: Bade, K. J./Bommes, M./Oltmer, J. (Hrsg.): Nachholende Integrationspolitik - Problemfelder und Forschungsfragen (IMIS-Beiträge Nr. 34). Osnabrück. 57-89

Die Bundesregierung (2007): Der Nationale Integrationsplan. Neue Wege - Neue Chancen. Berlin: Presse- und Informationsamt der Bundesregierung

Diehl, C./Friedrich, M./Hall, A. (2009): Jugendliche ausländischer Herkunft beim Übergang in die Berufsausbildung: Vom Wollen, Können und Dürfen. In: Zeitschrift für Soziologie, Jg. 38, H. 1. 48-67

Eymard-Duvernay, F. (2004): Économie politique de l'entreprise. Paris.

Eymard-Duvernay, F. (2008): Justesse et justice dans les recrutements. In: Formation Emploi, H. 101. 55-69.

Granovetter, M. (1995): Getting a Job. A Study of Contacts and Careers. Second Edition. Chicago.Holzer, H. J. (1987): Informal Job Search and Black Youth Unemployment. In: The American Economic Review, Jg. 77 , H. 3. 446-452

Hupka, S./Stalder, B. E. (2004): Die Situation junger Migrantinnen und Migranten beim Übergang Sek I/Sek II. In: Schweiz. Konferenz der Gleichstellungsbeauftragten (Hrsg.): Achtung Gender. Ausbildungsverhalten von Mädchen und jungen Frauen. Trends und Tipps. Zürich/Buchs. 79-94

Imdorf, Ch. (2005): Schulqualifikation und Berufsfindung. Wie Geschlecht und nationale Herkunft den Übergang in die Berufsbildung strukturieren. Wiesbaden.

Imdorf, Ch. (2007): Individuelle oder organisationale Ressourcen als Determinanten des Bildungserfolgs? Organisatorischer Problemlösungsbedarf als Motor sozialer Ungleichheit. In: Schweizerische Zeitschrift für Soziologie, Jg. 33, H. 3. 407-423

Imdorf, Ch. (2008): Migrantenjugendliche in der betrieblichen Ausbildungsplatzvergabe - auch ein Problem für Kommunen. In: Michael B./Krüger-Potratz, M. (Hrsg.): Migrationsreport 2008. Fakten - Analysen Perspektiven. Frankfurt a.M. 113-158

Jenkins, R. (1986): Racism and Recruitment. Managers, Organisations and Equal Opportunity in the Labour Market. Cambridge.

Kirschenman, J./Neckerman, K. M. (1991): "We'd Love to Hire Them, But...": The Meaning of Race for Employers. In: Jencks, C./Peterson, P. E. (Hrsg.): The Urban Underclass. Washington. 203-232

Lee, G./Wrench, J. (1983): Skill Seekers - black youth, apprenticeships and disadvantage. Leicester.

March, J. G./Simon, H. A. (1958): Organizations. New York.

Meyer, Th. (2009): Wer hat, dem wird gegeben: Bildungsungleichheit in der Schweiz. In: Suter, Ch./Perrenoud, S./Levy, R./Kuhn, U./Joye, D./Gazareth, P. (Hrsg.): Sozialbericht 2008. Die Schweiz vermessen und vergleichen. Zürich. 60-81

Moser, U. (2004): Jugendliche zwischen Schule und Berufsbildung. Eine Evaluation bei Schweizer 
Grossunternehmen unter Berücksichtigung des internationalen Schulleistungsvergleich PISA. Bern: h.e.p. Verlag

Mühlemann, S./Wolter, S. C./Fuhrer, M./Wüest, A. (2007): Lehrlingsausbildung - ökonomisch betrachtet. Ergebnisse der zweiten Kosten-Nutzen-Studie. Chur/Zürich

Pager, D. (2007): The Use of Field Experiments for Studies of Employment Discrimination: Contributions, Critiques, and Directions for the Future. In: The ANNALS of the American Academy of Political and Social Science, Vol. 609, H. 1. 104-133

Pager, D./Shepherd, H. (2008): The Sociology of Discrimination: Racial Discrimination in Employment, Housing, Credit, and Consumer Markets. In: Annual Review of Sociology, Jg. 34. 181-209

Phelps, E. S. (1972): The statistical theory of racism and sexism. In: American Economic Review, Jg. 62 September. 659-661

Quillian, L. (2006): New Approaches to Understanding Racial Prejudice and Discrimination. In: Annual Review of Sociology, Jg. 32. 299-328

Reskin, B. F. (2003): Including Mechanisms in Our Models of Ascriptive Inequality. In: American Sociological Reviev, Jg. 68, February. 1-21

Roscigno, V. J./Garcia, L. M./Bobbitt-Zeher, D. (2007): Social Closure and Processes of Race/Sex Employment Discrimination. In: The ANNALS of the American Academy of Political and Social Science, Vol. 609, H. 1. 16-48

Sayad, A. (2006): L'immigration ou les paradoxes de l'altérité. 1. L'illusion du provisoire. Paris: Raisons d'agir Éditions

Schaub, G. (1991): Betriebliche Rekrutierungsstrategien und Selektionsmechanismen für die Ausbildung und Beschäftigung junger Ausländer. Berlin

Seibert, H. (2005): Integration durch Ausbildung? Berufliche Platzierung ausländischer Ausbildungsabsolventen der Geburtsjahrgänge 1960 bis 1971. Berlin

Shih, J. (2002): "...Yeah, I could hire this one, but I know it's gonna be a problem": how race, nativity and gender affect employers' perceptions of the manageability of job seekers. In: Ethnic and Racial Studies, Jg. 25, H. 1. 99-119

Smith, A. (2006 [1790]): The Theory of Moral Sentiments. 6th ed.. Mineola: Dover Publications

Thévenot, L. (2001): Organized Complexity: Conventions of Coordination and the Composition of Economic Arrangements. In: European Journal of Social Theory, Jg. 4, H. 4. 405-425

Uhly, A./Granato, M. (2006): Werden ausländische Jugendliche aus dem dualen System der Berufsausbildung verdrängt? In: Berufsbildung in Wissenschaft und Praxis, H. 03/2006. 51-55

Waldinger, R./Lichter, M. I. (2003): How the Other Half Works. Immigration and the Social Organization of Labor. Berkeley.

Wieser, R./Dornmayr, H./Neubauer, B../Rothmüller, B. (2008): Bildungs- und Berufsberatung für Jugendliche mit Migrationshintergrund gegen Ende der Schulpflicht. Endbericht. Wien. 